\title{
Marginalisation et intégration dans la cité: éléments pour une lec- ture dynamique de la pensée politique de la République
}

\section{Marginalization and integration in the city: elements for a dyna- mic reading of the political thought of Plato's Republic}

\author{
ÉTIENNE HELMER \\ University of Puerto Rico (USA) \\ etiennehelmer@hotmail.fr
}

Recibido: 25/09/2016 - Aceptado: 15/09/2017

DOI: https://doi.org/10.20318/fons.2017.3857

\begin{abstract}
Résumé. On estime souvent que le cœur de la réflexion politique de Platon dans la République porte sur les conditions de possibilité de la cité juste. Une telle lecture se concentre alors sur l'institution des philosophes-rois, et sur l'organisation tripartite ou trifonctionnelle de la cité, avec à la clé un débat sur le caractère utopique ou non de cette construction politique. Sans être fausse, cette lecture est toutefois extrêmement partielle et réductrice en ce qu'elle occulte le point essentiel de la pensée politique de Platon dans ce dialogue: celle-ci ne se limite pas à l'analyse des conditions de possibilité de la cité juste, mais concerne plus généralement l'ensemble des processus d'intégration et de marginalisation de différentes catégories de population et de différentes fonctions requises pour édifier la cité. Trois arguments vont dans ce sens, que mon article présentera de façon détaillée. Premièrement, les difficultés d'élaboration du régime juste en raison des rapports tendus entre l'économie et la politique (livre II), ainsi que la dégradation des régimes sous l'effet du conflit entre ces deux sphères (livre VIII) invitent à voir dans la République une réflexion sur la politique comme dynamique socio-économique, plutôt qu'une description de la structure politique et économique statique sur laquelle se fonde la cité juste. Deuxièmement, les trois catégories fonctionnelles requises pour édifier la cité juste sont moins présentées comme des éléments prêts à y être intégrés et à y exercer leur tâche propre que comme des éléments dont le statut reste problématique et qui sont sujets à de nombreuses transformations affectant l'équilibre de l'ensemble de la cité. Cela se vérifie notamment à propos des philosophes, tant en ce qui concerne leur existence et leur éducation que leur préservation. Troisièmement, Platon ne se contente pas dans ce dialogue de décrire les trois catégories fonctionnelles qui composent la Kallipolis. Il y évoque aussi des catégories en apparence secondaires - les enfants sans talent, les mendiants, les incurables - dont il signale le degré d'intégration ou de marginalisation variable selon la forme du régime et les forces qui l'animent.
\end{abstract}

ПНГН/F O N S II (2017), 45-63

ISSN 2445-2297 www.uc3m.es/pege
É. HeLMER, Marginalisation et intégration dans la cité DOI https://doi.org/10.20318/fons.2017.3857 
Mots-clés: Cité, exclusion, intégration, marginalisation, Platon, République

\begin{abstract}
It is often assumed that the heart of Plato's political thought in the Republic addresses the conditions of possibility of the just city. Such a reading then concentrates mostly on the institution of the philosopher-kings, and the tripartite organization of the city. Without being wrong, this reading is however extremely partial and reductive as it obscures the main focus of Plato's political thought in this dialogue. Far from analyzing only the conditions of possibility of the just city, Plato's main concern deals with the processes of marginalization and integration of distinct population groups and functions in the city. Three arguments go in this direction. First, both the antagonistic relationships between economy and politics at the first stages of the city (Book II), and the progressive degradation of the regimes as a result of the conflict between these two spheres (Book VIII), invite us to read the Republic as a reflection on politics considered as a socio-economic dynamic, rather than a mere description of the static political and economic structure of the just city. Second, the three functional categories required to build this city are less presented as elements ready to be put at work, than as problematic elements, which are subject to many changes. This is especially true about the philosophers, with respect to their existence, education and preservation. Third, Plato also evokes secondary categories, in the just city and the ordinary cities as well - for instance, the children without talent, the beggars, the incurables - and he wonders about how and how far they can or must be integrated into or marginalized from the cities.
\end{abstract}

Keywords: City, exclusion, integration marginalization, Plato, Republic

\title{
Introduction
}

Pour la majorité de ses interprètes, la République de Platon porte principalement sur l'analyse des conditions de possibilité d'une cité juste, dont le philosophe décrirait les fondements et la structure. Leur lecture se concentre alors le plus souvent sur les points fondamentaux suivants: du côté des fondements, le principe de la fonction propre, les philosophes-rois et les grands principes éducatifs des citoyens et des gouvernants; du côté de la structure, l’organisation tripartite de la cité: les gardiens philosophes gouvernent, les auxiliaires défendent, les producteurs produisent ${ }^{1}$. Cette lecture est à la fois structurale, et statique ou dogmatique. Structurale au sens où elle décrit l'architecture fonctionnelle de la cité juste en rappelant comment chaque groupe contribue à l'édification et à la perpétuation de la cité par l'exercice de sa

Je remercie le Programme FIPI du Decanato de Estudios Graduados e Investigación de l'Université de Porto Rico (États-Unis) pour son aide matérielle dans l'élaboration de cet article.

${ }^{1}$ Par exemple: «In Plato's Republic, Socrates creates a regime in speech that attempts to incorporate justice into its orderly structure, where philosophers rule, guardians guard, and workers work», SAXONHOUSE 2009, p. 353. 
fonction spécifique. Statique ou dogmatique au sens où, bien qu'elle tienne compte des différentes étapes de la construction de la cité - de la première cité purement économique du livre II à la Kallipolis achevée - elle fait de cette dernière un idéal figé: elle réduit la réflexion de Platon à un programme ou à une série de principes pour édifier la cité juste, en occultant en général aussi bien les tensions qui perturbent son édification que tout ce qui l'ébranle et la détruit. Par exemple, les points de friction entre l'économie et la politique qui sont au cœur d'une partie du livre II ne sont presque jamais évoqués². De même, les livres VIII et IX dans lesquels Platon analyse comment la cité juste se défait et se transforme progressivement en différents régimes dégradés, sont souvent tenus pour secondaires et négligeables, voire confus et vains ${ }^{3}$. Quand les interprètes les évoquent, ce n'est bien souvent que sous l'angle psychologique, en vertu de l'analogie entre l'âme et la cité qui court dans toute la République 4 . Ceux qui proposent un commentaire politique des régimes dégradés ont tendance à n'étudier que la démocratie, pour des raisons évidentes d'écho à notre modernités.

Prise dans son ensemble, cette interprétation ne rend pas justice à la nature de la réflexion politique de Platon dans la République. Elle n'est pas fausse mais partielle et réductrice, parce qu'elle fige une pensée dont l'objet est en mouvement. La réflexion que Platon mène dans ce dialogue porte en effet, c'est ma thèse, sur les mouvements d'intégration et de marginalisation de différentes fonctions, sphères d'activité et catégories de population envisagées dans leur devenir politique. Par intégration et marginalisation dans la République, j'entends l'ensemble des mouvements et leurs diverses causes (psychologiques, économiques, politiques) qui contribuent respectivement à conduire la cité vers son unité harmonieuse ou, au contraire, à y propager la désunion et le conflit. Ces mouvements peuvent être plus ou moins poussés et prendre des formes plus ou moins radicales, l'exclusion, par exemple, étant un cas extrême de marginalisation.

Ces jeux dynamiques se produisent à trois niveaux. D'abord, au niveau des grandes sphères fonctionnelles qui participent à l'édification de la cité. Par exemple, on a peu noté les tensions que Platon observe entre la tendance destructrice de

${ }^{2}$ Pour une lecture du livre II de la République faisant état de ces tensions, voir HeLmer 2010, pp. 34-64.

${ }^{3}$ Négligeable: LaNe 2006 n'évoque pas ce passage dans son article de synthèse. Confus: ANNAS 1981, p. 294; et en partie Rosen 2005, p. 309 à propos du passage de l'aristocratie à la timocratie. Vain: PAPPAS [1995] 2005, pp. 120-123. Il y a quelques exceptions cependant: par exemple MEULDER 2013, qui évoque notamment les processus d'exclusion à l'œuvre en oligarchie.

${ }^{4}$ Par exemple BlÖSSNER 2007.

${ }^{5}$ Ainsi Santas 2010, pp. 158-186; SCHOFIELD 2006, pp. 106-107; il faut toutefois reconnaitre qu'il consacre quelques pages aux autres régimes (258-264), mais sans entrer dans le détail de la causalité complexe faisant passer d'un régime à l'autre. Pour une analyse plus détaillée de ces livres, voir Helmer 2006; et Hitz 2010, pp. 103-131. 
l'économie quand elle est livrée à elle-même, et la puissance d'intégration et d'unification qu'il prête à la politique droite. Les équilibres instables, sans cesse recomposés, entre ces deux sphères, se soldent par la marginalisation de la politique droite au profit d'une gestion de la cité qui n'a de politique que le nom. Ces rapports de force et ces jeux de composition entre les deux domaines rendent raison en partie des transformations des régimes, et justifient le caractère dynamique de la lecture que je propose. Ce caractère vaut aussi pour les groupes qui composent la cité juste. La République ne se contente pas de présenter trois catégories fonctionnelles parfaitement intégrées dans un ensemble économique, social et politique unifié : elle évoque surtout leur caractère problématique, soit tout ce qui rend difficile leur existence même, ainsi que tout ce qui la menace et la corrompt. C'est notamment le cas avec le philosophe: la proposition socratique des «philosophes-rois», à laquelle on tend souvent à réduire le contenu politique de la République, est moins une thèse qu'un problème, puisque ce sont les difficultés d'éducation et de préservation du naturel philosophe qui occupent la majeure partie de l'ouvrage. Enfin, quelle que soit la cité considérée - la Kallipolis comme les autres ${ }^{6}$ - Platon mentionne toujours diverses catégories de population qui en débordent le cadre, et dont il se demande dans chaque cas quelle place leur assigner: enfants sans talent, individus de santé fragile, incurables sur les plans éthique et psychologique, mendiants. Là encore, la réflexion de Platon porte moins sur la décision à prendre regardant ces catégories que sur les critères et les motifs expliquant le caractère problématique de leur intégration dans la cité.

La République contient ainsi selon moi davantage une réflexion sur la dynamique politique, sociale et économique et ses effets sur le degré d'unité de toute cité, que la description de la structure statique de la cité juste. Loin de se limiter à énoncer des règles pour la formation de cette seule cité, Platon y examine plutôt comment se fait et se défait toute cité en général. C'est l'esquisse de cette nouvelle lecture d'ensemble de la réflexion politique de la République, qui rende justice à son caractère dynamique, que je propose de dessiner ici en revenant plus en détail sur les trois points évoqués dans le paragraphe précédent.

I. Faire et défaire la cité: forces économiques et forces politiques en tension

L'élaboration de la cité en paroles entamée au livre II de la République est souvent présentée comme une succession d'étapes conduisant d'une cité économique, d'abord saine (II, 369a-372e) puis fébrile (II, 372e-374e), à une cité politique achevée culminant dans l'annonce des «philosophes-rois» (V, 473c-e), sans solution de continuité entre ces étapes, comme s’il s'agissait d'un empilage de ni-

\footnotetext{
${ }^{6}$ Par exemple: «[The] existence [of political knowledge] is a premise of the construction of the just city in Plato's central political work, the Republic, and lies at the root of his most famous political doctrine, the rule of philosopher-kings», HiTz 2009, p. 367 (je souligne).
} 
veaux fonctionnels s'ajustant parfaitement les uns aux autres. De même, ceux qui se penchent sur la dégradation des régimes à partir de la cité aristocratique des philosophes-rois aux livres VIII et IX tendent à s'arrêter à la description de chaque étape avec le type psycho-politique qui la caractérise, en fonction de la ou des valeurs suprêmes auxquelles il obéit. Or qu'il s'agisse de l'élaboration ou de la détérioration de la cité juste, dans tous les cas, l'impasse est faite sur ce que Socrate et ses interlocuteurs mettent pourtant en avant: les forces en tension à l'œuvre dans le passage d'une étape à une autre, dont le travail va soit dans le sens d'une intégration des fonctions et des hommes, soit dans le sens de leur marginalisation par rapport à la structure politique. Or c'est le jeu de ces forces, économiques et politiques notamment, qui forme une part essentielle du questionnement philosophique du dialogue et qui fait de la pensée politique de Platon une réflexion dont l'objet est plus dynamique et processuel que statique et arrêté.

\section{1. Faire la cité ou comment intégrer l'économique dans le politique}

La naissance de la cité en paroles au livre II se caractérise par une tension entre, d'un côté, la tendance à la multiplication et à l'extension des fonctions économiques qui lui donnent le jour, avec leurs soubassements anthropologiques que sont les besoins et les appétits, et, de l'autre, la nécessaire limitation que Socrate tente d’imprimer à la cité pour garantir son unité politique. Cette tension est repérable dès les premières lignes du récit de la naissance de la cité:

\footnotetext{
Socrate: Eh bien, dis-je, une cité, je crois, vient à être pour autant que chacun de nous se trouve

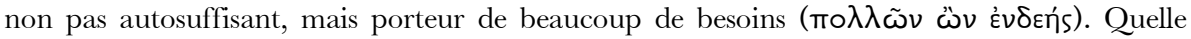
autre origine crois-tu qu'il y ait à la fondation d'une cité?

Glaucon: Aucune autre, dit-il.

Socrate: Ainsi donc, un homme en prend un second pour le besoin d'une chose, et un troisième pour le besoin d'une autre chose; et comme ils ont beaucoup de besoins (по $\lambda \lambda \tilde{\omega} \nu$ $\delta \varepsilon o ́ \mu \varepsilon v o ı)$, ils rassemblent beaucoup d'hommes en un seul lieu d'habitation, associés pour se rendre ser-

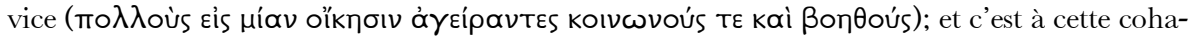
bitation que nous avons donné le nom de cité (Rép. II, 369b-c).
}

À la grande quantité des besoins sur laquelle Socrate insiste dans ce court passage s'oppose l'unicité du territoire et l'idée de mise commun qui sont essentielles à sa conception de la cité comme ensemble politique. Socrate jette là les bases d'une opposition qui, si elle n'est pas encore conflictuelle, va toutefois le devenir très rapidement, comme le montrent les deux points suivants?

Premièrement, Socrate pose comme motif de l'échange des objets produits par les divers métiers le fait que chacun «croit que ce sera meilleur pour soi-même» (369c). Or l'articulation entre ce que chacun estime être le meilleur pour soi-même et ce qui

\footnotetext{
${ }^{7}$ Pour une analyse détaillée de ce passage, voir HeLmer 2010, pp. 36-64.
} 
est véritablement le meilleur en soi, ordonné à l'Idée du Bien et devant valoir pour toute la cité, est un thème central de la réflexion politique de la République ${ }^{8}$. Tandis que, la suite va le montrer, les besoins tendront vers une particularisation et une extension croissantes sous la forme d'appétits dès lors conflictuels entre eux et avec le commun de la cité, la politique philosophique devra travailler à la détermination et à la mise en œuvre d'un Bien qui soit à la fois en mesure de satisfaire les aspirations des individus et celle du tout. Les autres politiques, elles, laisseront libre jeu à ce conflit et même le nourriront parce que leurs gouvernants n'atteignent pas l'Idée du Bien.

Deuxièmement, Socrate rappelle que ce qui fonde la cité c'est «notre besoin» (í

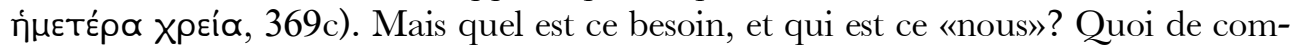
mun en effet entre Socrate qui maîtrise ses appétits, et le tyran aux appétits démesurés? Sur fond de l'expérience anthropologique commune de la nécessité, est laissée en suspens la question de savoir comment, à partir de besoins fondamentaux génériquement identiques, se développent des appétits de nature et d'ampleur particuliers, et dans quelle mesure cette diversité peut être compatible avec un «nous» politique unitaire. La question que pose Socrate - «comment la cité suffira-t-elle à produire tant de choses?» (369d) - à propos des besoins fondamentaux que sont la nourriture, le logement, «l'habillement et les choses de ce genre», pointe la tension entre unité politique et multiplicité économique, tension qu'explicitera le passage de la cité saine, aux besoins variés mais limités, à la cité malade (II, 372d-e). À Glaucon insatisfait de la première cité qu'il qualifie de «cité de cochons» parce qu'elle manque de

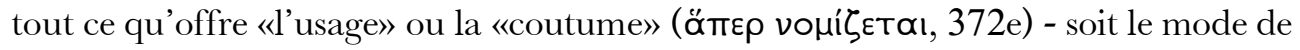
vie courant des Athéniens -, Socrate répond en effet en acceptant volontiers

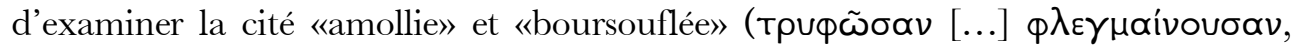
372e) parce que telle est bien, pour lui et Platon, la difficulté politique des cités ordinaires: savoir contrer les effets destructeurs du développement économique, placé sous le signe de la multiplicité indéfinie, sur l'unité de la cité. Tous les produits qui viennent gonfler la polis y pénètrent en effet «sous toutes sortes de formes» (ह̌k $\alpha \sigma \tau \alpha$

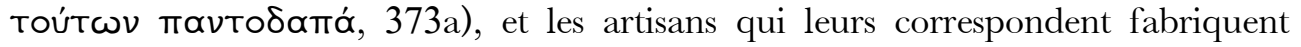

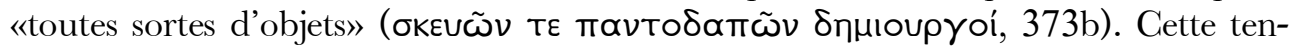
dance rend nécessaire ce qui était superflu (373a) et façonne un désir «d'acquisition

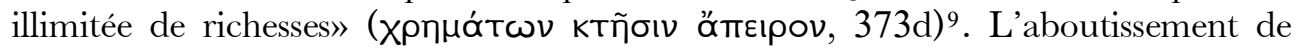
cette démultiplication n'est autre que la destruction potentielle de la «cité» comme entité politique dans l'épreuve inévitable de la guerre - il faudra bien faire la guerre

${ }^{8}$ L'exemple le plus évident est sans doute le cas des gardiens évoqués en IV, 419a-421c, en particulier 421b-c: «Socrate: Il faut donc examiner si nous voulons instituer des gardiens en visant le but suivant: que le plus grand bonheur possible leur échoie à eux; ou s'il faut envisager ce but pour la cité entière, et examiner si le bonheur lui advient à elle».

${ }^{9}$ Sur le désir de richesse chez Platon, voir Helmer 2016. 
pour trouver les ressources devant satisfaire autant de besoins (373d-e). C'est ce qui justifie l'entrée en lice des gardiens (373e-374e), et qui ouvre à la question générale de savoir quelle politique et quel personnel politique peuvent être en mesure de concilier l'exigence d'unité politique d'un côté, et la tendance démultiplicatrice et destructrice des économies livrées à elles-mêmes de l'autre ${ }^{10}$.

\section{2. Défaire la cité: la politique marginalisée par l'économique}

Le jeu de l'intégration et de la marginalisation des grandes sphères fonctionnelles indispensables à l'édification de la cité, l'économique et la politique principalement, est également au centre de l'analyse des régimes dégradés au livre VIII de la République. Leur succession s'explique en effet en partie par l'incapacité des gouvernants à contrôler les forces centripètes de la sphère économique, dont ils alimentent la puissance destructrice par leur incapacité politique même. La sphère politique se trouve ainsi marginalisée par rapport à ce que doit être son lieu fonctionnel central, qu'occupent désormais des forces dont les manifestations collectives principales sont de type économique - une économie livrée de plus en plus à elle-même et faisant le jeu du désir de posséder déjà repéré au livre II comme étant à l'origine de la guerre. On peut en donner deux exemples flagrants: le régime aristocratique ou philosophique, et le régime oligarchique.

La marginalisation fonctionnelle de la sphère politique - et sa détérioration concomitante - commence dès le régime des philosophes-rois, pourtant fondé sur l'équilibre du rapport entre l'économique et le politique par leur commune intégration dans un ensemble unitaire. Dans la cité juste en effet, la séparation d'un groupe en charge de la production d'un côté, et d'un autre en charge du gouvernement de l'autre, va de pair avec leur mise en commun dans le cadre d'un système d'échange mutuel et complémentaire en vertu duquel le premier est «nourricier» du second, et le second «sauveur» du premier (Rép. V, 463a-b). Or, précise Socrate, «il est difficile que soit ébranlée une cité aussi cohérente. Mais puisque pour tout ce qui est né, il y a corruption, cette structure non plus ne pourra se maintenir à jamais, mais se dissoudra» (Rép. VIII, 546a). Ce principe très général, rarement souligné et encore moins commenté ${ }^{11}$, se prête à une lecture dynamique plus précise en termes d'équilibre instable des forces ou des dimensions fonctionnelles de la cité. En d'autres termes, même l'intégration réussie de l'économique et du politique dans le

${ }^{10}$ Un passage du livre IV (422d-423c) reviendra sur cette tension entre unité de la cité, et désunion sociale et économique entre ses membres quand les ressources y sont trop inégalement réparties. Socrate déclare: «Chaque cité [ordinaire] est un grand nombre de cités, et non une cité [...]. Il y en a d'abord deux, quelle que soit la cité, en guerre l'une contre l'autre: celle des pauvres et celle des riches. D'autre part, dans chacune de ces deux-là, il y en a un grand nombre [...]», Rép. IV, 422e-423a.

${ }^{11}$ Seule Hitz le mentionne, mais elle se contente de signaler que Socrate se réfère à un "principe général», Hitz 2010, p. 109. 
meilleur des régimes reste fragile. Elle requiert une vigilance et une restauration constantes, pourtant impossibles à mettre en œuvre indéfiniment. Le résultat de l'intégration des fonctions dans la cité juste ne doit donc pas être perçue comme un ensemble figé et statique, qui s'effriterait peu à peu comme une maison tombe progressivement en ruines, mais comme un équilibre momentané de forces en tension, qui travaillent chacune dans leur sens jusqu'à démembrer ce qu'elles étaient pourtant parvenu à construire en s'appuyant l'une sur l'autre.

L'une des conséquences de cette rupture d'équilibre est le décentrement fonctionnel de la politique, et par là sa détérioration, au profit de préoccupations économiques censées en tenir lieu mais qui, en forçant la sphère politique à se reconfigurer, donnent lieu à un nouvel équilibre d'ensemble lui aussi très fragile et précaire. C'est ce que souligne Socrate dans le passage suivant:

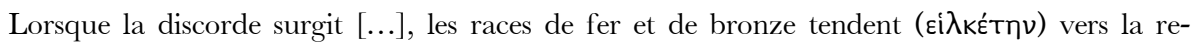
cherche de la richesse, de la possession de la terre, des habitations, de l'or et de l'argent, tandis

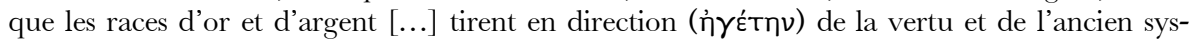
tème. Comme elles se font violence et se tendent les unes contre les autres (

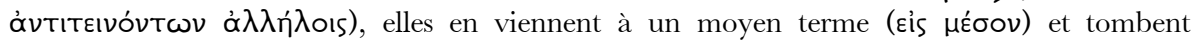
d'accord pour privatiser, en les répartissant, la terre et les maisons.

Rép. VIII, 547b-c

Tout l'intérêt de cet extrait tient à la dynamique qu'il évoque et que traduit le vocabulaire de la tension des forces tirant en des sens opposés. La discorde en question, qui désigne soit un conflit psychique interne aux gardiens, soit un conflit entre les gardiens et les producteurs dans la cité, soit les deux à la fois ${ }^{12}$, montre dans tous les cas que le monde de l'économie domestique refait surface et s'impose conjointement comme modèle d'organisation «politique» - les guillemets étant de rigueur puisqu'il s'agit d'une politique dégradée et marginalisée, ne méritant donc pas pleinement son nom - et comme source de diffusion d'une économie plus générale faisant désormais le jeu du privé au détriment du commun de la cité. Ce changement se signale dans la rupture de l'échange fonctionnel mutuel qui caractérisait la cité juste: désormais, les gouvernés ne sont plus considérés comme les nourriciers des gouvernants mais comme leurs domestiques et leurs esclaves, comme si la cité n'était plus qu'un grand oikos scindé entre maîtres et serviteurs (Rép. VIII, 547c). La solution de compromis qui est trouvée - placer eis meson la terre et les maisons pour les partager - ne saurait être que provisoire: une fois affranchi de toute tutelle politique authentique, le désir d'avoir ou de posséder qui anime les «races de fer et de bronze» va se propager sous des formes diverses et conduire à la dégradation des régimes, soit à la relégation croissante de la politique et à sa détérioration au profit d'une écono-

\footnotetext{
${ }^{12}$ Sur l'interpénétration des vocabulaires psychologiques et politiques, voir LEAR 1992, pp. 184215.
} 
mie elle-même déréglée. L'expression eis meson, qui désigne à l'origine la répartition du butin après la bataille et la distribution de la parole dans les assemblées selon le principe de l'isonomie ${ }^{13}$, ne renvoie ainsi qu'à une mise en commun de façade ou précaire parce qu'elle est concurrentielle, ce qui s'affirmera encore plus dans les régimes suivants. Là encore, l'équilibre nouveau des forces est instable et de courte durée.

Passons à l'oligarchie ${ }^{14}$. La valeur publiquement reconnue dans ce régime à la richesse se signale dans le portrait de l'homme oligarchique: pour s'éviter les déceptions et l'ingratitude par quoi se sont soldés les efforts et les risques liés aux responsabilités publiques de son père timocrate, l'homme oligarchique cherche à édifier laborieusement une fortune, «accumulant les richesses à coup de petites économies et à force de travail» (Rép. VIII, 553c). La reconnaissance qu'il cherche à s'attirer ne doit provenir que de sa richesse et des efforts qu'elle lui a coûtés:

[L'homme oligarchique] oblige le principe rationnel à ne faire rien d'autre que calculer et rechercher les moyens, à partir de moins d'argent, d'en avoir plus, et il force l'élément d'ardeur à n'admirer et à n'honorer que la richesse et les riches, et à s'enorgueillir de ce que sa réputation ne soit fondée sur rien d'autre que la possession de richesses et de tout autre bien propre à y contribuer (Rép. VIII, 553d).

Un tel type d'homme entérine ainsi définitivement le passage d'un monde où le commun primait encore sur le particulier à un monde où c'est le particulier et le privé qui façonnent ce qu'on peut difficilement appeler encore un monde commun. Déjà en germe chez les timocrates qui trouvaient des motifs de dépense «pour euxmêmes» (Rép. VIII, 550d) tout en faisant publiquement de l'honneur leur vertu cardinale, la configuration du régime oligarchique accélère la promotion au niveau public d'une valeur dont la satisfaction ne relevait jusqu'alors que de la jouissance privée parce qu'elle traduisait un désir d'accaparement néfaste pour l'ordre commun de la cité. Désormais, le désir de devenir «le plus riche possible» (Rép. VIII, $554 \mathrm{a}$; 555b) l'emporte sur toute autre considération, au point que le degré de richesse devient le critère de l'exercice du pouvoir (Rép. VIII, 555c).

Cette soif de richesse ouvertement déclarée est propice à des pratiques économiques qui consacrent la division de la cité, et dont le principe général est que la richesse des uns se construit sur la misère des autres. Ainsi du prêt à intérêt, dont Socrate dénonce l'usage et les conséquences:

Ceux qui commandent dans cette constitution politique n'exercent leur commandement, je pense, que parce qu'ils ont beaucoup acquis; ils ne cherchent pas à contrôler les jeunes qui deviennent indisciplinés, pour les empêcher de dépenser leurs biens et leur éviter la ruine. Leur but est, en achetant leurs biens et en leur prêtant à intérêt, de devenir encore plus riches et plus

\footnotetext{
${ }^{13}$ Voir Detienne 1967, pp. 131-153.

${ }^{14}$ Voir Meulder 2013, pp. 89-98.
} 
considérés. [...] Ainsi dans les oligarchies, c'est en les négligeant et en tolérant l'indiscipline que les dirigeants réduisent parfois à la pauvreté des hommes qui n'étaient pas dépourvus de qualités par leur naissance (Rép. VIII, 555c-d).

L'oligarchie promeut une économie de l'endettement, c'est-à-dire de l'appauvrissement et de l'asservissement. Elle offre ainsi l'exemple le plus clair d'un régime où, sous l'effet du décuplement du désir de posséder, la perversion de la vie politique va de pair avec celle de l'économie. Car cette soif de richesse met en péril l'économie de subsistance dont toute cité a besoin, menaçant ainsi la cité elle-même. Contrairement à ce que préconisait le principe de spécialisation individuelle formulé au livre II, chacun est en effet incité ici à remplir plusieurs fonctions: désormais «l'agriculture, les affaires, la guerre sont, dans cette constitution politique, aux mains des mêmes personnes» (Rép. VIII, 551e-552a), ce qui porte préjudice à la fois à la vie politique - les dirigeants n'étant plus les meilleurs mais les plus riches - et à l'efficacité économique - l'essentiel n'étant plus de bien produire et de produire le nécessaire, mais de gagner le plus d'argent possible. L'analyse de l'oligarchie signale donc là encore que c'est le jeu des transformations mutuelles de la politique et de l'économie dans une configuration globale nouvelle et en devenir qui intéresse Platon, et non la description d'une vignette figée dans un tableau systématique des régimes.

Loin d'empiler le politique sur l'économique dans la phase d'élaboration de la cité, et de les démembrer quand il s'agit d'étudier sa dégradation, Platon les présente bien plutôt dans leurs rapports évolutifs mutuels pour inviter à réfléchir sur la façon dont ces deux sphères étroitement liées produisent de l'intégration ou de la marginalisation politique d'ensemble. Cette dynamique, toutefois, ne se limite pas aux grandes sphères d'activité de toute cité. Elle vaut aussi, dans le cas particulier de la Kallipolis, pour chacun des groupes fonctionnels qui la composent.

II. Dynamique des trois groupes fonctionnels dans la cité juste: le cas des philosophes, entre intégration et marginalisation

De toutes les propositions politiques de la République, celle de faire les philosophes rois ou de rendre les rois philosophes pour remédier aux maux des cités et des hommes (Rép. V, 473c-e) est sans aucun doute la plus célèbre et celle qui, pour certains, résume ou du moins concentre l'essentiel du dialogue ${ }^{15}$. Si l'ensemble de la conversation tend certes vers elle comme vers le pivot sur lequel repose tout l'édifice de la Kallipolis, elle doit toutefois être prise moins comme un résultat ou une thèse que comme un problème, contrairement à l'interprétation la plus courante. Ce caractère problématique, qui place le philosophe au centre d'un jeu d'intégration et de marginalisation, est repérable dans deux grands moments du dialogue. L'un, en amont de l'annonce des «philosophes rois», signale les difficultés d'éducation du na-

${ }^{15}$ Voir la note 6. 
turel philosophe, soit les résistances qui se posent à son intégration politique en raison du caractère très spécifique des qualités qu'il doit posséder. L’autre, en aval, signale toutes les forces de marginalisation et de dégradation qui œuvrent contre lui et contre l'exercice de la fonction centrale qui devrait lui revenir ${ }^{16}$. Ces deux passages étayent ainsi l'hypothèse du caractère dynamique de la pensée politique de Platon dans ce dialogue. Examinons-les successivement.

\section{1. Comment intégrer le philosophe-roi?}

Lue de près, l'élaboration des philosophes-rois se révèle pleine de rebondissements qui en dénoncent le caractère problématique. Tout d'abord, si l'attribution de la fonction de gardien à un groupe séparé s'inscrit dans le droit fil du principe de la fonction propre, sur la base duquel a été organisée la répartition des fonctions productives en vue de rendre les échanges économiques «plus faciles» et plus efficaces (Rép. II, 370a-c), le cas de ces gardiens est toutefois présenté comme un peu à part: la nature bien particulière de leur fonction, souligne Socrate, rend sa séparation d'avec les autres encore plus impérative. Le raisonnement de Socrate est le suivant: les autres techniques sont déjà difficiles et requièrent d'être exécutées séparément; or «ce qui concerne la guerre, n'est-il pas encore plus important que cela soit bien accompli?» (Rép. II, 374c); donc la fonction des gardiens doit d'autant plus être séparée des autres. Bref, "plus la fonction des gardiens est importante, plus elle aurait besoin d'être dégagée le plus possible des autres fonctions, et plus elle aurait besoin aussi qu'on y applique un art et un soin extrêmes» (Rép. II, 374d-e), Socrate semblant anticiper ici la distinction future des gardiens en auxiliaires d'un côté, et en gouvernants philosophes de l'autre (Rép. III, 414b). Ce que ce passage souligne de façon assez appuyée n'est toutefois pas tant un principe organisationnel à suivre qu'un danger latent et radical, celui de la confusion des fonctions, qui sera reformulé après l'énoncé du «noble mensonge»: «Dès lors que [les gardiens] eux-mêmes auront acquis un terrain privé, des maisons et des monnaies en usage, ils seront administrateurs de maisons et cultivateurs au lieu d'être des gardiens, et ils deviendront les maîtres hostiles et non plus les alliés des autres citoyens» (Rép. III, 417a-b). L'exigence de séparation fonctionnelle à propos des gardiens est ainsi avancée et soulignée parce qu'elle s'adosse à un péril majeur qui, on va le voir, n'est pas qu'une hypothèse théorique.

Les gardiens sont en effet tout particulièrement exposés à ce risque de confusion parce qu'il est, pour une part, intrinsèquement lié aux qualités qui les définissent. La façon dont ces qualités sont présentées au moment de brosser leur portrait consiste moins à en faire la liste qu'à montrer comment chacune d'elle, avancée comme une

\footnotetext{
${ }^{16}$ On ne peut suivre M. Meulder qui soutient que «[...] l'exclusion (de la cité) n'existe pas dans le régime philosophique», MEULDER 2013, p. 108.
} 
solution à une difficulté antérieure, devient à son tour la source d'une nouvelle difficulté théorique et pratique. Ainsi, dès l'énoncé de la première qualité requise pour les gardiens, à savoir qu'ils devront être pleins de cœur pour être «viril» ou courageux et bien défendre la cité (Rép. II, 375a-b), Socrate soulève le problème qui en découle: «de quelle façon éviter que les gardiens se comportent avec sauvagerie les uns envers les autres et envers le reste des citoyens, si telles sont leurs natures?» «Par Zeus, dit [Glaucon], ce n'est pas facile» (Rép. II, 375b). La qualité suivante, celle de la douceur, requise précisément pour équilibrer le caractère plein de cœur, est présentée à son tour moins comme une solution au problème que Socrate vient de soulever que comme la source d'une nouvelle difficulté, celle de savoir comment peuvent coexister des contraires dans un même naturel: «où trouverons-nous une façon d'être à la fois douce et pleine de cœur [...]? Ceci ressemble à une situation impossible et s'il en est ainsi, il est impossible que se forme un bon gardien» (Rép. II, 375cd). Or à peine l'existence d'un exemple d'animal - le chien - possédant les deux caractères opposés est-elle avancée par Socrate pour sortir de cette impasse que, de nouveau, surgit une difficulté: celle de son éducation. Avant d'y venir, notons que la référence au chien semble servir uniquement à montrer que la coexistence des contraires dans une même nature n'est pas impossible (Rép. II, 375d-376c). Mais cela suffit-il à conclure qu'elle sera possible chez d'autres êtres, et en particulier chez l'homme? L'éducation pourra-t-elle être imiter efficacement la nature? Plutôt qu'une solution, la référence au chien ouvre encore une fois à une nouvelle série de questions, qui rendent la possibilité du philosophe-roi rien moins qu'évidente.

Dernier passage qui met en avant le caractère problématique du philosophe-roi, celui qui porte donc sur son éducation, en particulier celle qui concerne la musique et la gymnastique (Rép. III, 410a-412b). Comme avec les caractères - la douceur et le tempérament plein de cœur - la question est de savoir comment unir des qualités ou des exigences contraires pourtant requises par la fonction qui les appelle et par le naturel qui lui correspond. La pratique exclusive de la gymnastique risque en effet de rendre plus brutal qu'il ne faut, tandis qu'à l'inverse, la pratique exclusive de la musique risque de rendre plus mou qu'il ne le faut (Rép. III, 410d). Après avoir décrit les éducations excessives dans les deux sens, Socrate conclut que la musique est requise pour attendrir l'élément plein de cœur, et la gymnastique pour raffermir l'élément philosophique et sa douceur, afin que les deux caractères s'harmonisent en l'âme (Rép. III, 411e-412a). Encore une fois, Socrate insiste moins sur ce qu'il faut faire que sur la description des risques d'une éducation déséquilibrée, comme si l'éducation qu'il est en train de préconiser était par avance fortement menacée.

Le pivot central de la République semble donc être moins le philosophe-roi que les questions et les problèmes qu'il soulève, souvent gommés dans la littérature secondaire au profit d'une annonce lue comme un énoncé directement applicable ou efficace. Ce que Platon fait voir, c'est bien plutôt que l'intégration du philosophe - 
c'est-à-dire de l'unique candidat légitime à l'exercice du pouvoir - est en soi la plus haute difficulté qui se pose dans la réflexion politique de ce dialogue. Les menaces qui pèsent sur lui et la description de sa corruption le confirment.

\section{2. Le philosophe à la marge}

Par ses qualités mêmes, le philosophe est exposé à un risque de marginalisation bien plus extrême que celui qui menace les autres membres de la cité, et qui a pour cause les dégradations pouvant affecter son naturel bien particulier. Pour expliquer ce phénomène, au livre VI, Socrate rappelle d'abord que le naturel philosophe «naît rarement et en petit nombre parmi les hommes» (Rép. VI, 491b). Or c'est la friction entre les qualités même de ce naturel (virilité, tempérance, etc.) et tous les prétendus biens valorisés dans les cités ordinaires - richesse, beauté, force du corps - qui explique cette dégradation. C'est qu'en effet les âmes les plus douées, quand elles ne trouvent pas la nourriture qui leur convient, c'est-à-dire «quand elles rencontrent une mauvaise pédagogie, deviennent exceptionnellement mauvaises» (Rép. VI, 491e). Éduqué au contact de la masse et des institutions collectives de la cité, le naturel philosophe se pervertit sous l'effet des jugements de valeur collectif dont il lui est très difficile de faire abstraction, surtout quand on le menace de mort (Rép. VI, 492b-d). Une autre forme de corruption se produit quand un jeune homme présentant toutes les qualités pour se livrer à la philosophie est sollicité par ses concitoyens pour s'occuper de leurs affaires. Il risque alors de s'emplir «d'un espoir démesuré, s'imaginant être à même de s'occuper à la fois des affaires des Grecs et de celles des Barbares, et là-dessus, ne va-t-il pas s'exalter, tout enflé de poses et prétentions vaines, au lieu de réfléchir?» (Rép. VI, 494c-d). Le philosophe devient alors l'objet d'un «conflit» dans la cité: si l'on tente de le ramener vers la philosophie, les hommes qui l'ont sollicité «ne vont-ils pas tout dire et tout faire, à la fois auprès de lui et auprès de celui qui cherche à le convaincre [de s'adonner à la réflexion] pour l'empêcher d'en être capable, aussi bien en complotant contre lui en privé qu'en lui faisant affronter des procès en public?» (Rép. VI, 494d-e). La claire allusion au procès de Socrate et, à la fin de l'allégorie de la caverne, à sa mort (Rép. VII, 517a), sont autant d'indices que la place du philosophe dans la cité est contrariée par des forces de marginalisation ou d'exclusion inhérentes au rapport entre la cité empirique et le philosophe, et qui l'empêchent d'exercer la fonction qui devrait lui revenir. La précarité des philosophes qui redescendent dans la caverne est telle qu'il fau-

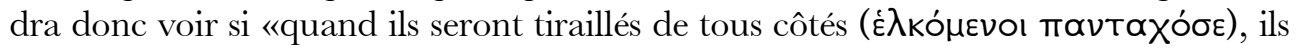
resteront fermes ou se laisseront ébranler» (Rép. VII 539e-540a): comme dans le texte du livre VIII cité plus haut qui évoquait le conflit opposant la race de fer à la race d'or, c'est le vocabulaire de la tension et du tiraillement qui est évoqué pour dire l'équilibre très fragile de la position des philosophes dans la cité. 
À de très rares exceptions près - celui qui, en exil, est préservé des influences corruptrices et peut pratiquer la philosophie; celui qui peut le faire parce qu'il vit dans une petit cité et en dédaigne les affaires communes; ou encore celui qui vient à la philosophie après avoir abandonné un autre art (Rép. VI, 496b) - tout, dans les cités, s'oppose donc à l'épanouissement du naturel philosophe, tout le conduit inévitablement à se pervertir (Rép. VI, 497b), ce qui laisse le champ libre aux usurpateurs qui revendiquent l'exercice de la philosophie mais en réalité la déshonorent (Rép. VI, 495b-497b). Objet d'une forte marginalisation, voire d'une exclusion totale, le philosophe est encore une fois présenté comme un être problématique plutôt que comme une panacée. La métaphore de Socrate pour décrire la posture du philosophe dans la cité ordinaire est elle-même à comprendre moins comme une «solution» durable que comme un équilibre instable: «on se tient tranquille et on s'occupe de ses propres affaires, comme quand dans la tempête, lorsque nuages de poussière et tornades d'eau sont soulevés par le souffle du vent, on s'abrite au pied d'un muret» (Rép. VI, 496d). Mais combien de temps le muret pourra-t-il résister à la force des éléments?

Derrière l'annonce des philosophes-rois, Socrate pointe donc en réalité autre chose: comment, dans les cités ordinaires, devenir philosophe et comment le rester? Comment intégrer la fonction politique dans ses conditions optimales d'exercice et comment éviter sa marginalisation? Dans les deux cas, c'est la stabilité de l'édifice politique qui est en jeu, et un ensemble de forces en tension qui font l'objet de la réflexion de Platon.

\section{La place des sans place}

Les mouvements d'intégration et de marginalisation qui me semblent donner à l'objet de la réflexion politique de Platon dans la République son caractère dynamique concernent aussi diverses catégories de population, dont il signale la place problématique aussi bien dans la Kallipolis que dans les cités ordinaires. Comme si toute polis était, par nature, confrontée la question de ses frontières politiques et à la nécessité d'exclure un certain nombre de catégories pour en intégrer d'autres et continuer ainsi à exister comme polis, ces catégories étant définies par la configuration politique, économique et sociale, propre à chaque type de cité. Les enfants de plus de 10 ans dans la Kallipolis, et les mendiants dans la cité oligarchique en offrent de bons exemples.

\section{1. Les enfants de plus de 10 ans}

La mesure peut sembler anecdotique tant Socrate l'évoque rapidement, au moment de conclure l'exposé des conditions requises pour que la Kallipolis voie le jour: «ceux qui dans la cité, dis-je, se trouveront avoir plus de dix ans, ils [les gardiens] les

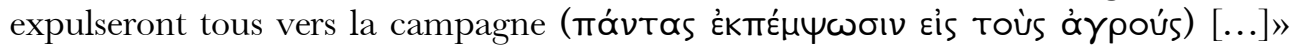


(Rép. VII, 540e-541a). Ce passage a donné lieu à deux interprétations opposées. Pour les uns, Socrate recommande de mettre à l'abri les enfants de cette classe d'âge pour les préserver de l'influence corruptrice des mœurs de la cité17. Pour d'autres, au contraire, il s'agit de mettre à l'écart de la nouvelle cité tous ceux qui sont déjà trop âgés pour se laisser modeler par son nouveau système éducatif parce qu'ils portent les traces indélébiles de l'éducation défaillante dispensée dans la cité antérieure ${ }^{18}$. Cette seconde interprétation a l'avantage d'être plus cohérente que la première avec les mesures éducatives présentées dans les livres antérieurs: en particulier, c'est dès l'état de nourrisson que les enfants des gardiens seront élevés en commun pour briser l'attachement parental et filial particulier, au profit du sentiment d'appartenance à la grande famille des gardiens (Rép. V, 457c-d); de même, c'est dès le plus jeune âge que l'éducation réformée en vue du Beau devra faire son œuvre dans les âmes (Rép. III, 400e) et qu'il faudra, dans la mesure du possible, entrainer et emmener les enfants à la guerre (Rép. V, 466e-467a). Ajoutons aussi que cette interprétation est consonante avec un autre passage de la République où Socrate préconise de dérober aux regards les enfants difformes ou nés en dehors des âges autorisés de procréation pour les gardiens (V, 460c-461c). Si donc la seconde interprétation semble la plus plausible, quel sens politique lui donner, au-delà de son enjeu éducatif?

Plutôt qu'une «injustice» requise pour fonder la cité juste et dont on voit mal comment Platon ou Socrate pourrait s'en accommoder ${ }^{19}$, on peut y lire une réflexion sur ce que «faire la cité» veut dire. Platon semble indiquer par là que le tout de la cité juste, envisagé non pas comme un modèle purement théorique mais dans le versant pratique de sa mise en œuvre, ne peut voir le jour sans un double jeu d'intégration et d'exclusion de certaines catégories de la population. Ce jeu à double sens s'exerce de différentes façons quand la cité se met en place. Ce peut être entre les trois groupes mêmes qui composent la Kallipolis, comme le montre la relégation des nouveau-nés dans la catégorie fonctionnelle qui leur convient (Rép. IV, 423c-d) et qui peut être distincte de celle où ils voient jour, au nom de l'intégrité de chacune d'entre elles et de leur intégration harmonieuse dans une cité une. Mais ce peut être aussi entre le dedans de la cité et son dehors, comme le signale aussi bien l'exemple des enfants de plus de 10 ans que celui de l'élimination des individus que Socrate nomme «incurables». Il n'est donc de tout de la cité que sur la base d'un principe de sélection et d'exclusion, que la République a en commun avec le Politique (308e309a) et les Lois (V, 735d-e $)^{20}$. Ce principe, qui semble animer la cité dans son être même, est toutefois plus flagrant dans les cités empiriques, que Socrate présente

\footnotetext{
${ }^{17}$ Par exemple SHEPPARd 2009, p. 125.

${ }^{18}$ Par exemple Rosen 2005, pp. 140 et 301; et ReEve 2006, p. 180.

${ }^{19}$ Rosen 2005, p. 301.

${ }^{20}$ Sur ce principe et sur les incurables, voir HeLmer 2017.
} 
comme des machines à marginaliser, voire à exclure. C'est notamment le cas avec les mendiants dans le régime oligarchique.

\section{2. Les mendiants en oligarchie}

Quand il ne désigne pas par métaphore ceux qui sont avides de richesses sous l'effet de la domination des appétits en leur âme (Rép. VII, 520a-521b), le mendiant au sens propre est pour Platon un symptôme, c'est-à-dire à la fois un signe et un effet. Son existence témoigne et résulte nécessairement d'un défaut dans l'organisation économique, sociale et politique des cités empiriques. Ce défaut est celui de la cupidité collective, de cet appétit de richesse (philokhrèmatia) dans lequel Platon voit la source du mal politique, puisque c'est son inévitable résurgence dans le régime des philosophes qui précipite dans un malheur croissant les cités et les hommes, jusqu'à les conduire à la tyrannie. C'est en particulier dans le régime oligarchique évoqué au livre VIII (550c-553a) que les mendiants font leur apparition, sous l'effet d'un mécanisme économico-politique lâchant la bride à des forces de marginalisation et d'intégration particulièrement violentes.

La présence dans ce régime de mendiants (Rép. VIII, 552c-e), qui sont contraints de solliciter l'assistance des autres pour se procurer le nécessaire, est le résultat de la violation par les dirigeants du principe de la fonction propre posé au livre II, et selon lequel chaque membre de la cité ne doit exercer qu'une seule et unique fonction, sous peine de troubler l'harmonie, la justice et l'unité de la cité. Or en plus de l'exercice du pouvoir, les dirigeants oligarques se lancent aussi dans les affaires pour gagner de l'argent. L’intensité de leur désir de richesse n'a d'égal que leur goût de la dépense, de la dissipation de leurs ressources, cause et effet du principe selon lequel dans cette cité, où la richesse est la valeur suprême, tout peut s'acheter et se vendre, au point de rendre certains habitants «excessivement riches et les autres tout à fait pauvres». Comme de faux-bourdons, «fléaux de l'essaim», ces faux-dirigeants sont le fléau de la cité. Lorsqu'ils sont, à la différence des faux-bourdons des ruches, pourvus d'un aiguillon, ils deviennent des malfaiteurs, des prédateurs effroyables qui acquièrent leur richesse au prix de la misère des autres; lorsqu'ils en sont dépourvus, une fois leurs richesses dilapidées, ils se trouvent réduits à la mendicité. Ce schéma appliqué aux gouvernants semble surtout valoir pour les différencier des gouvernés qui, dans la cité oligarchique, sont à peu près tous réduits à mendier, comme en témoigne l'échange suivant entre Socrate et Adimante:

Socrate: Il est donc évident, dans une cité où tu vois des mendiants ( $\pi \tau \omega \times \supset u ́ s)$, qu’il se trouve dissimulé quelque part dans ce lieu des voleurs, des coupeurs de bourses, des pilleurs de temples, et des artisans de tous les méfaits de ce genre.

Adimante: C'est évident, dit-il.

Or dis-moi: dans les cités gouvernées de façon oligarchique, ne vois-tu pas que se trouvent des mendiants ( $\pi T \omega X$ Xús)? 
$\mathrm{Si}$, dit-il, et peu s'en faut qu'ils ne le soient tous, à l'exception des dirigeants.

Ne devons-nous pas penser alors, dis-je, qu'il s'y trouve aussi de nombreux malfaiteurs avec aiguillons, que les autorités veillent à contenir par la force?

$\mathrm{Si}$, nous devons le penser, dit-il.

N'affirmerons-nous pas alors que c'est à cause du manque d'éducation, de la mauvaise façon de les élever, et de la mauvaise institution du régime politique, que de tels hommes sont venus à naître?

Si, nous l'affirmerons (Rép. VIII, 552d-e).

Cette omniprésence des mendiants en oligarchie est confirmée par la présentation du type psychologique oligarchique, qui valorise la richesse plus que tout et la thésaurise (553a3-555b2). Celui-ci cherche à acquérir de l'argent par tous les moyens, en particulier par des pratiques économiques qui, comme le prêt à intérêt, favorisent la naissance des deux sortes de faux-bourdons, malfaiteurs et mendiants $(555 \mathrm{e} 3-$ 556a3). Le mendiant signale donc à la fois l'existence de son frère, le malfaiteur, et la valeur suprême accordée à la richesse par tout un régime, de la part de ses gouvernants comme de ses gouvernés. Dans les deux cas, il est la preuve d'une criante imperfection politique associée à une éducation imparfaite qui en est autant la cause que l'effet, et qui se traduit par la marginalisation économique d'une grande partie des citoyens, ainsi que par une apparente intégration des politiques. «Apparente» car si l'emploi d'un instrument financier comme le prêt à intérêt leur permet pour un temps de consolider leur pouvoir, une bonne partie d'entre eux n'en finissent pas moins par se marginaliser eux-mêmes : plus préoccupés de leur richesse que de leur tâche propre, ils la dilapident et deviennent eux-mêmes des mendiants. La cité semble ainsi produire des équilibres instables et précaires, destinés à se modifier et à la disloquer sous l'effet de la reconfiguration des éléments et des forces qui la composent tant bien que mal à un moment donné.

Mis à part le remède radical et général qui permettrait de remettre les cités et les hommes sur le chemin de la justice, à savoir l'instauration d'un régime gouverné par les philosophes (Rép. V, 473c-d), Platon mentionne aussi un remède plus spécifique et plus directement applicable pour éradiquer la mendicité régnant en oligarchie, remède qui joue comme force d'intégration sociale, économique et politique à la fois: la réforme du prêt au moyen d'une loi prescrivant «de conclure la plupart des contrats volontaires aux risques du prêteur», ce qui éviterait les enrichissements excessifs que les faux-bourdons malfaiteurs réalisent au détriment des autres citoyens (VIII, 556c-e) ${ }^{21}$.

\section{Conclusion}

Les analyses qui précèdent ne prétendent pas avoir épuisé les arguments en faveur de la lecture dynamique de la République que je défends. Elles veulent seule-

\footnotetext{
${ }^{21}$ On trouve un projet similaire dans les Lois V, 742c et XI, 916d-e.
} 
ment suggérer à grands traits, sur la base de quelques exemples saillants, que l'interprétation la plus admise de la réflexion politique de Platon dans la République tend à gommer ce caractère dynamique, en faisant indûment abstraction des mouvements et des forces de marginalisation et d'intégration qui s'y déploient de différentes façons et à différents niveaux. Une étude plus détaillée serait requise pour valider cette lecture, ainsi que pour montrer comment, à l'inverse, la lecture la plus admise de ce dialogue a pu influencer une partie de l'histoire de la philosophie politique occidentale.

On peut se demander si l'interprétation dynamique que je défends peut valoir pour l'ensemble de la réflexion politique de Platon au-delà de la seule République, en particulier pour les Lois qui, elles aussi, proposent un modèle de cité juste. Il semble que le caractère dynamique observé dans la République et fondé sur la construction problématique de la Kallipolis soit absent des Lois: dans ce second dialogue prédomine en effet le souci d'élaborer un code législatif répondant à des exigences éthiques, politiques et anthropologiques déterminées. Ce n'est pas que la réflexion politique de Platon ait changé de nature, c'est plutôt qu'elle présente un autre aspect dès qu'on aborde la mise en œuvre effective des principes et des réflexions sur lesquels elle repose.

\section{Bibliographie}

Annas, J. (1981), An Introduction to Plato's Republic, Oxford.

Blössner, N. (2007), «The city-soul analogy», dans F. Ferrari (ed.), The Cambridge Companion to Plato's Republic, Cambridge, 345-385.

Detienne, M. (1967), Les Maîtres de vérité dans la Grèce archaïque, Paris.

Helmer, É. (2006), «Histoire, politique et pratique aux livres VIII-IX de la République», dans M. Dixsaut (éd.), Études sur la République, I, Paris, 149-168.

Helmer, É. (2010), La Part du bronze. Platon et l'économie, Paris.

Helmer, É. (2013), «Philosophies grecques du mendiant», Les Cahiers philosophiques, 134, 3-16.

Helmer, É. (2016), «Platon et le désir de richesse: psychologie, économie et politique», dans É. Helmer (éd.), Richesse et pauvreté chez les philosophes de l'Antiquité, Paris, 197-220.

Helmer, É. (2017), «Aux frontières de la cité. Les incurables de Platon», Philosophie antique, $17,125-148$.

Hitz, Z. (2009), «Plato on the Sovereignty of Law», dans R.K. Balot (ed.), A Companion to Greek and Roman Political Thought, London, 367-381.

Hitz, Z. (2010), «Degenerate Regimes in Plato's Republic», dans M.L. McPherran (ed.), Plato's Republic. A Critical Guide, Oxford, 103-131.

Lane, M. (2006), «Plato's Political Philosophy: the Republic, the Statesman, and the Laws», dans M.L. Gill, P. Pellegrin (eds.), A Companion to Ancient Philosophy, London, 172180. 
Larivée, A. (2016), «Si Socrate devenait cité: pauvreté, souci des richesses et soin de l’âme dans l'Apologie et la République», dans É. Helmer (éd.), Richesse et pauvreté chez les philosophes de l'Antiquité, Paris, 221-250.

Lear, J. (1992), «Inside and Outside the Republic», Phronesis, 37, 184-215.

Lötter, H.P.P. (2003), «The significance of poverty and wealth in Plato's Republic», South African Journal of Philosophy, 22, 189-206.

Meulder, M. (2013), «L’exclusion au livre VIII de la République de Platon», Revue belge de philologie et d'histoire, 91, 89-112.

Pappas, N. (2005), Routledge Philosophy Guidebook to Plato and the Republic, New York.

Reeve, C.D.C. (2006), Philosopher-Kings. The Argument of Plato's Republic, Indianapolis.

Rosen, S. (2005), Plato’s Republic. A Study, Yale.

Santas, G. (2010), Understanding Plato’s Republic, London.

Saxonhouse, A.W. (2009), «Freedom, Tyranny, and the Political Man: Plato's Republic and Gorgias, a Study in Contrasts», dans R.K. Balot (ed.), A Companion to Greek and Roman Political Thought, London, 353-366.

Schofield, M. (2006), Plato. Political Philosophy, Oxford.

Sheppard, D.J. (2009), Plato's Republic, Edinburgh. 